\title{
Welcome to PCR London Valves 2014
}

\author{
Martyn Thomas ${ }^{1}$, MBBS, MD, FRCP; Stephan Windecker ${ }^{2 *}$, MD, FESC \\ 1. Guy's \& St Thomas' Hospitals NHS Foundation Trust, London, United Kingdom; 2. Department of Cardiology, Swiss \\ Cardiovascular Center Bern, Bern, Switzerland
}

Welcome to PCR London Valves 2014. This is now the fifth edition of this Heart Valve meeting and we are delighted it is now fully established and very much a part of the calendar of worldwide interventional meetings. Indeed, PCR London Valves has become the largest meeting in the world dedicated to transcatheter valvular interventions designed for the Heart Team. Once more the meeting is being held in the heart of London in the Queen Elizabeth Conference Centre, right next to the Houses of Parliament, "Big Ben" and Westminster Abbey. We are proud that we are able to provide such an iconic venue for our course.

This supplement is designed to reflect the content of the 2014 edition of PCR London Valves. This year we are introducing an exciting new feature, with the introduction of "The Great Valve Debate". This will build on the concept pioneered at EuroPCR, but here it will be specifically targeted to the field of transcatheter valvular interventions. The topic for the 2014 debate was selected by you, the interventional cardiology community, and is entitled "Does the improvement of TAVI outcomes justify treatment of low-risk patients?". This theme reflects the rapid pace at which this field continues to evolve in view of important technological advances.

In this supplement, we have invited Pieter Kappetein and Alain Cribier to provide - from a surgical and interventional point of view, respectively - the differing perspectives concerning this issue which is widely debated throughout Europe. Within this same discussion is the question as to whether the TAVI procedure should be simplified. This will be extensively discussed during the meeting and one personal viewpoint is provided here in the supplement by Antonio Colombo's group.

We now have multiple TAVI devices available in Europe and Heart Teams are now faced with the decision as to whether they will use only one or multiple TAVI devices within a single institution. Here again, there are dedicated sessions within PCR London Valves 2014 related to this topic, and Mohamed Abdel-Wahab, the lead author of the first randomised trial comparing the outcomes of two TAVI devices recently published in JAMA, discusses the CHOICE trial in this supplement.

Live cases remain the bedrock of the sessions in the Main Auditorium, and this year there will be three Live Transmission Centres: St Thomas' Hospital in London, UK; Bern Hospital in Bern, Switzerland; and the Heart Centre in Bonn, Germany. These centres will use a variety of different devices in order to illustrate the decision making regarding the choice of these devices for different valve interventions. We are very grateful to Georg Nickenig and his colleagues in Bonn, Germany, for agreeing to join us (along with the Heart Teams at St Thomas' Hospital and Bern) as a Live Centre to try to achieve this aim.

This year, 2014, saw a number of transcatheter mitral valve implantation (TMVI) programmes finally beginning their firstin-human studies. This is an emerging field, which is quite early in its development and is being jointly explored by both cardiac surgeons and cardiologists. During the meeting, many sessions will be devoted to mitral valve anatomy and mitral valve interventions, something which is reflected in the number of manuscripts on both TMVI and mitral valve repair within this supplement. This is going to be a long journey, and the community will need to carefully explore appropriate patient selection as well as the risks and benefits of transcatheter mitral valve repair and replacement techniques.

The technique of transcatheter valvular intervention is a complex one. It requires a Heart Team with multiple skills. An integral 
element to this is specialised imaging. Learning these skills is not easy and requires multiple modalities. By definition, teaching these skills is equally challenging. During the course three teaching aids and techniques will be introduced to further support the learning process on this topic. On the Sunday preceding the meeting, "The Sunday Imaging Day" will use the forum of didactic lectures and "hands on" workshops to give Heart Teams, including imaging specialists, a comprehensive understanding of the use of all imaging modalities including echo, CT and MRI for TAVI and mitral interventions. The second teaching aid is the "valve-in-valve App" which facilitates both aortic and mitral valve-in-valve procedures. These procedures require extensive preprocedural knowledge of the surgical bioprosthesis used, including the various size measurements and the "true" internal diameter. The "valve-in-valve App" will be highlighted during the meeting in valve-in-valve sessions, and a manuscript describing the valve-in-valve technique and the use of the App is included in this supplement. Finally, the "TAVI Valve Atlas" will be introduced during PCR London Valves 2014. The OCT Atlas has been highly successful and this latest interactive
TAVI software package will follow a similar multimodality interactive format. Use of the TAVI Valve Atlas will be demonstrated in a dedicated session during PCR London Valves 2014.

PCR London Valves 2014 will once again be a highly interactive practical meeting designed "by" and dedicated "for" Heart Teams. Much of the programme will involve open and friendly discussion of cases and abstracts submitted by the interventional community. The meeting's "ethos" is that of the sharing of knowledge rather than didactic lecturing by the faculty. The main arena will once more be dedicated to Live Case transmissions. In 2015, PCR London Valves will have a one-year break from London and will go "on the road" to Berlin, entitled "PCR London Valves in Berlin 2015". This is for two reasons: one, because the European Society of Cardiology Congress is taking place in London in September 2015; and two, because we feel the outstanding and international contribution of Germany to the field of TAVI should be recognised!

We hope this supplement will be a ready and useful additional reference for the interactive sessions that are planned and welcome you all, once again, to PCR London Valves...enjoy the meeting. 